\title{
Efecto del Aliskireno sobre la Angiogénesis en Modelo de Membrana Alantocoriónica (MAC) de Pollo
}

\author{
Effect of Aliskiren on Angiogenesis in Chick \\ Embryo Chorioallantoic Membrane (CAM) Assay
}

\author{
Raúl de la Fuente E.*; Carlos Rosas C.**; Marcela Fuenzalida B.; Héctor Rodríguez B.*; \\ Ignacio Roa ${ }^{* * * * * * *, * * * * * *}$ \& David Lemus A.*
}

DE LA FUENTE, E. R.; ROSAS, C. C.; FUENZALIDA, B. M.; RODRÍGUEZ, B. H. ; ROA, I. \& LEMUS, A. D. Efecto del aliskireno sobre la angiogénesis en modelo de membrana alantocoriónica (MAC) de pollo. Int. J. Morphol., 34(4):1191-1196, 2016.

RESUMEN: La acción antiangiogénica de los inhibidores del receptor de angiotensina II (ARA II), ha sido documentada previamente, sin embargo, no ha sido descrita la relación entre angiogénesis e inhibidores directos de la renina (DRIs), los cuales participan regulando el sistema renina-angiotensina-aldosterona (SRAA). El objetivo fue demostrar el efecto antiangiogénico de aliskireno, un DRI, en membranas alantocoriónicas (MAC) de pollo, para lo cual fueron instilados aliskireno y enalapril sobre MAC en distintas concentraciones para realizar su comparación posterior. En secciones histológicas seriadas se registró el número de vasos sanguíneos presentes en $9000 \mu \mathrm{m}^{2}$ bajo microscopio de luz a máximo aumento, y se realizó análisis estadístico utilizando ANOVA y el test de Tukey para demostrar posibles diferencias. Los receptores tratados con aliskireno, en ambas concentraciones utilizadas, presentaron menor densidad vascular, en comparación con los controles, siendo ésta estadísticamente significativa a mayor concentración. Aliskireno en concentraciones altas tiene un efecto antiangiogénico en un modelo experimental de MAC. Este hallazgo plantea la necesidad de estudios posteriores, dada la proyección que podría tener el uso inhibidores directos de la renina. A partir de estos resultados, se podría pensar en la factibilidad del uso de aliskireno para la modulación de la angiogénesis en diversas enfermedades crónicas no transmisibles.

PALABRAS CLAVE: Angiogénesis; Membrana alantocoriónica; Aliskireno; Enalapril.

\section{INTRODUCCIÓN}

La angiogénesis es definida como la neoformación de vasos sanguíneos a partir de vasos preexistentes (Schmidt \& Carmeliet, 2010). Durante este complejo fenómeno se establece: proliferación y migración celular, reclutamiento celular, activación de diversas moléculas (de adhesión celular, interleuquinas, factores de crecimiento, etc.) y activación de múltiples vías de señales celulares (Santulli, 2013). El regulador angiogénico más relevante es el factor de crecimiento vascular endotelial tipo A (VEGF-A) (Gavalas et al., 2013). Esta citoquina es expresada fisiológicamnete en tejidos tales como placenta y cuerpo lúteo, y se une a dos receptores con actividad tirosina kinasa: VEGFR-1 y VEGFR-2, siendo este último el más importante en su acción, aun cuando VEGF-A se une con una afinidad 50 veces mayor al primero (Goel \& Mercurio, 2013). La acción de VEGF-A se lleva a cabo principalmente sobre las células del endotelio vascular, aumentando su permeabilidad de diversas maneras; aumentando el transporte intracelular a través de organelos y vesículas y reorganizando las uniones cadherina/catenina deshaciendo las uniones estrechas intercelulares (Bates, 2010), además de estimular la producción de activadores del plasminógeno y sus inhibidores (Pepper, 1997), así como de colagenasa intersticial (Arvelo \& Cotte, 2006).

El sistema renina-angiotensina-aldosterona (SRAA) es una cascada enzimática compleja, que se activa sea frente a una disminución de la presión arterial o de los niveles de sodio en los túbulos renales (Ferrão et al., 2014). Se inicia con la formación de renina por las células del aparato yuxtaglomerular del riñón, la que al ser liberada al torrente sanguíneo, cataliza la transformación del angiotensinógeno,

* Programa de Anatomía y Biología del Desarrollo. Facultad de Medicina, Universidad de Chile, Santiago, Chile.

** Departamento de Ciencias Morfológicas, Facultad de Ciencia, Universidad San Sebastián, Santiago, Chile.

*** Unidad de Morfología, Departamento de Ciencias Básicas Biomédicas, Facultad de Ciencias de la Salud, Universidad de Talca, Chile.

***** Programa de Doctorado en Ciencias Morfológicas, Facultad de Medicina, Universidad de La Frontera, Temuco, Chile.

****** Becario CONICYT-PCHA/Doctorado Nacional/2015-21150235. 
producido en el hígado, en angiotensina I, la que a su vez es transformada en angiotensina II, por acción de la enzima convertidora de angiotensina (ACE), a nivel pulmonar. La angiotensina II se une a dos receptores principales, denominados receptores de angiotensina II tipo 1 (AT1) y tipo 2 (AT2). La unión de angiotensina II a AT1 estimula la producción en la corteza suprarrenal de aldosterona, mineralocorticoide que produce reabsorción de agua y sodio en los túbulos renales, aumentando de este modo tanto el volumen intravascular como el volumen minuto. Si bien no se conoce con certeza la acción del receptor AT2, tendría una acción opuesta a AT1. La acción angiogénica de angiotensina II conlleva una transactivación del receptor del factor de crecimiento epidérmico (EGF), que a su vez involucra una fosforilación del receptor VEGFR-1 (Ferrara \& Kerbel, 2005).

Actualmente, se sabe que en el SRAA existe otra vía, en que participan el heptapéptido angiotensina (1-7) (Harding et al., 1992; Santos et al., 2003) y el homólogo de enzima convertidora de angiotensina denominado ACE2. La formación de angiotensina (1-7) se da principalmente mediante la acción de ACE2 sobre angiotensina II, (Vickers et al., 2002). Se ha descrito también la unión de angiotensina (1-7) al receptor asociado a proteína $\mathrm{G}$ Mas, por lo que su acción es independiente de la unión a los receptores AT1 o AT2. De este modo, se tienen dos ejes en el SRAA: ACE/angiotensina II/AT1 y ACE2/ angiotensina (1-7)/Mas.

El eje ACE2/angiotensina (1-7)/Mas tiene un efecto tanto antiangiogénico como antiproliferativo, lo que se debería a una inhibición tanto en la fosforilación de ERK como en la activación de COX-2, ambas moléculas involucradas en procesos de proliferación celular (Passos-Silva et al., 2013). Asimismo, se ha observado que angiotensina (1-7) regula negativamente la actividad angiogénica de las células endoteliales (Calnan \& Brunet, 2008).

Los inhibidores directos de renina (DRI, del inglés Direct Renin Inhibitors) producen un bloqueo completo del SRAA, evitando efectos colaterales como el aumento compensatorio en los niveles de angiotensina II, o el aumento en la actividad de la renina plasmática. El DRI más importante es el aliskireno, fármaco autorizado por la FDA para el tratamiento de la hipertensión en 2007 (Morales Olivas \& Yago, 2009).

No existe literatura que describa la respuesta angiogénica en vasos sanguíneos, luego de la administración directa de un DRI. El objetivo el presente estudio fue describir la acción directa de aliskireno sobre la angiogénesis en un modelo in vitro de membrana alantocoriónica (MAC).

\section{MATERIAL Y MÉTODO}

Se utilizaron 90 huevos de pollo White Leghorn fertilizados, obtenidos del Instituto de Salud Pública (ISP), Chile, los que fueron lavados con un algodón embebido en agua destilada, desinfectados con alcohol yodado y marcados en su superficie superior, para ser ubicados en bandejas en posición horizontal para que el blastodisco se ubique en posición dorsal y mantenidos en cámara de incubación esterilizada a una temperatura de $37,8^{\circ} \mathrm{C}$ en una atmósfera humidificada.

En el día 3, la superficie superior del huevo fue perforada de él extraidos $2 \mathrm{ml}$ de albúmina, de modo que la MAC en desarrollo se separara de la cáscara. Posteriormente esta fue cerrada con papel celofán y se abrió una segunda ventana de 2,5 x $2 \mathrm{~cm}$ en la marca previamente señalada, exponiéndose los vasos sanguíneos subyacentes. Las burbujas generadas a partir de la succión de albúmina fueron eliminadas según lo descrito por Toledo et al. (2010). Finalmente, se selló la ventana con cinta adhesiva transparente y los receptores fueron devueltos a la incubadora, donde permanecieron una semana. A partir de este momento, los receptores fueron permanentemente monitoreados, con el fin de retirar aquéllos que no sobrevivieron o que no se desarrollaron.

Al día 10, fue instalado de un disco de metilcelulosa (Millipore $^{\mathrm{TM}}$ ) de $5 \mathrm{~mm}$ de diámetro, esterilizado, sobre la superficie de la MAC, en la cual se instilaron las siguientes soluciones: Grupo Control ( $\mathrm{n}=12)$ : agua bidestilada; Grupo 1 $(\mathrm{n}=14)$ : agua bidestilada + enalapril 2,03 $\mu \mathrm{M}$; Grupo 2 ( $\mathrm{n}=14)$ : agua bidestilada + enalapril 8,12 $\mu \mathrm{M}$; Grupo $3(\mathrm{n}=16)$ : agua bidestilada + aliskireno 54, $37 \mu \mathrm{M}$; Grupo $4(\mathrm{n}=16)$ : agua bidestilada + aliskireno 108,74 $\mu \mathrm{M}$.

Al día 13, se removieron las MAC y fijadas en formalina al $10 \%$ durante 24 horas, posteriormente lavadas con agua (Michel et al., 2011) y teñidas con HematoxilinaEosina (H-E).

Se determinó la densidad microvascular, contando la totalidad de vasos sanguíneos por $9000 \mu \mathrm{m}^{2}(30 \mu \mathrm{m} \times 300$ $\mu \mathrm{m})$, en un microscopio óptico PrimoStar (Carl Zeiss, Göttingen, Germany), con un aumento de 400x y ocular ubicado a una distancia de $600 \mu \mathrm{m}$ del filtro. Se efectuaron conteos en cada uno de los grupos de trabajo, obteniéndose valores muestrales del número de capilares sanguíneos (Fernández et al., 2014; Rosas et al., 2014).

El análisis estadístico se realizó a través de ANOVA de una vía con Test de Tukey. Se consideró estadísticamente significativo un P-value <0,05. Para la realización de los gráficos se utilizó el programa Graph Pad Prism ${ }^{\circledR} 5$. 


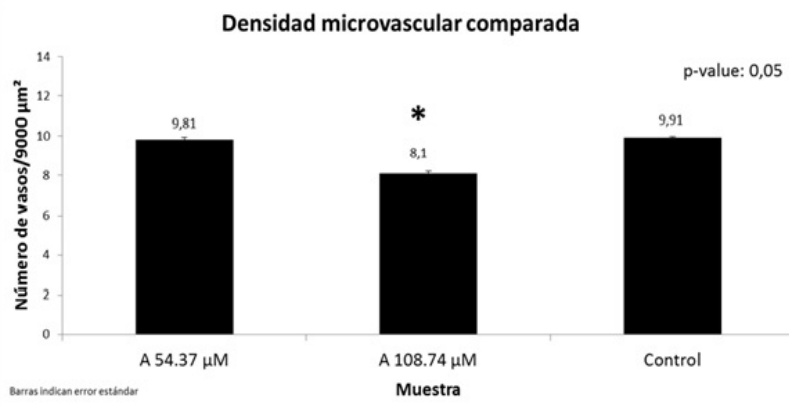

Fig. 1. Aliskireno disminuye la densidad microvascular. La diferencia es estadísticamente significativa solo a la concentración máxima estudiada.

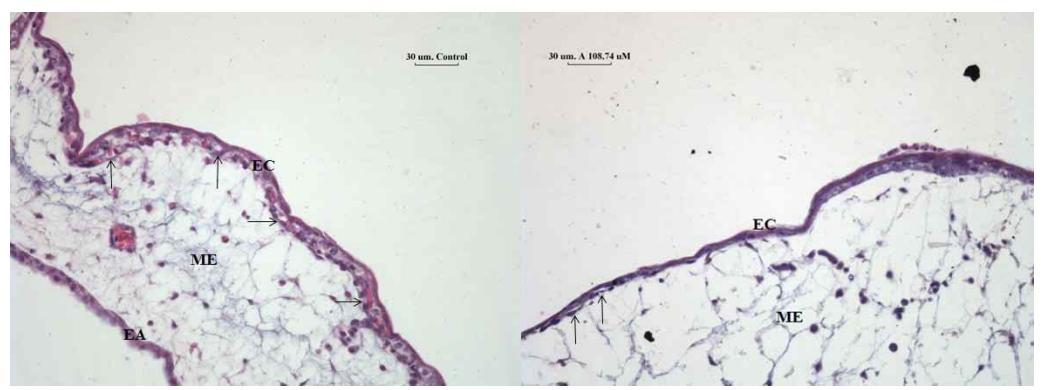

Fig. 2. MAC de pollo, Grupo Control y Grupo 4 (agua bidestilada + aliskireno $108,74 \mu \mathrm{M})$, en donde se aprecia una caída en la migración de vasos sanguíneos de neoformación hacia el corion (flechas). EC: Epitelio coriónico, ME: Tejido mesenquimático, EA: Epitelio alantoides. H/E. Tamaño barra $30 \mu \mathrm{m}$.

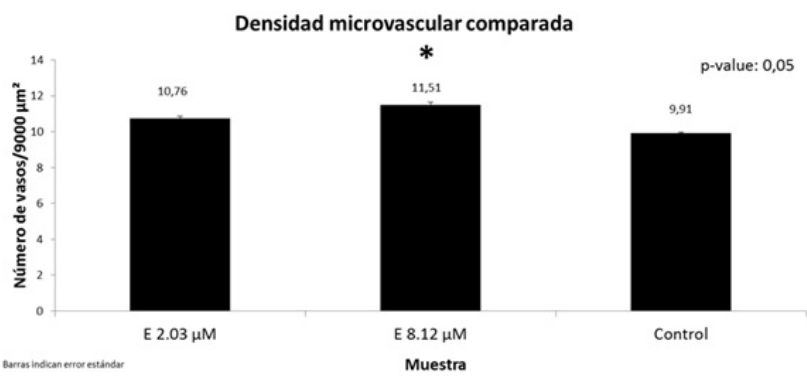

Fig. 3. Enalapril aumenta la densidad microvascular. La diferencia es estadísticamente significativa solo a la mayor concentración máxima estudiada.

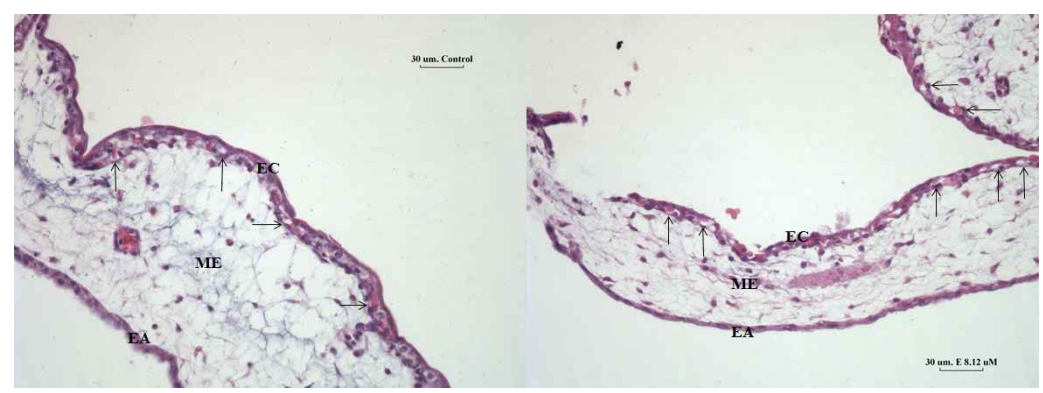

Fig. 4. MAC de pollo, Grupo Control y Grupo 2 (agua bidestilada + enalapril 8,12 $\mu \mathrm{M})$ en donde se ve una mayor migración de vasos sanguíneos de neoformación hacia el corion (flechas). EC: Epitelio coriónico; ME: Tejido mesenquimático, EA: Epitelio alantoideo. H/E. Tamaño barra $30 \mu \mathrm{m}$.

\section{RESULTADOS}

Del total inicial de 90 receptores, solo 66 fueron utilizados para el análisis debido a pérdidas durante los ensayos. Finalmente los grupos quedaron distribuidos de la siguiente forma: Grupo Control ( $\mathrm{n}=12)$, Grupo $1(\mathrm{n}=12)$, Grupo $2(n=12)$, Grupo $3(n=16)$ y Grupo $4(n=14)$.

La administración de aliskireno en las concentraciones utilizadas, produjo inhibición de la angiogénesis en modelos de MAC, siendo este efecto estadísticamente significativo respecto a los controles solo en aquellos sujetos instilados con alta concentración de este fármaco $(\mathrm{P}<0,05)$ (Fig. 1).

Como consecuencia de lo anterior, se observó también, en huevos receptores de aliskireno en altas concentraciones, una menor migración de vasos sanguíneos neoformados hacia el corion, en relación a los controles (Fig. 2).

Por su parte la administración de enalapril en las concentraciones estudiadas, produjo un efecto proangiogénico en modelos de MAC de pollo, encontrándose diferencia estadísticamente significativa en relación a los controles solo en aquellos sujetos que recibieron este fármaco en la concentración máxima utilizada (P <0,05) (Fig. 3).

\section{DISCUSIÓN}

En relación al efecto de aliskireno sobre la angiogénesis, la acción inhibitoria observada es estadísticamente significativa solo al administrarse en la mayor concentración utilizada. Este hallazgo contradice los primeros trabajos descritos en la literatura, que señalan al aliskireno como un regulador positivo de fenómenos proangiogénicos en modelos de ratas (Pöss et al., 2010; Rusai et al., 2011). Sin embargo, además de las diferencias metodológicas, dichos estudios se basaron en parámetros indirectos para evaluar la actividad angiogénica, tales como el número de células positivas para el VEGFR2, o el índice angiopoietina 1 / angiopoietina 2. Así como los resultados de Chang et al. 
(2015), los cuales relataron que aliskireno induce neovasculogénesis dosis dependiente a través de mecanismos relacionados VEGF/SDF-1 $\alpha$ en un modelo de ratones diabéticos. Resultados similares a los encontrados por Desjarlais et al. (2105), en donde aliskireno además incrementó la cantidad y actividad de las células progenitoras endoteliales derivadas de médula ósea. Los resultados obtenidos en el presente estudio confirman la hipótesis de trabajo relativa al rol antiangiogénico de aliskireno, lo que sí es consistente para lo descrito previamente en relación a la acción de los ARA II, con la consecuente inhibición del SRAA. Una posible explicación estaría dada por una mayor sensibilidad del eje ACE/ angiotensina II/AT1 a la inhibición, lo que generaría un predominio relativo de la acción del eje ACE2/angiotensina (1-7)/Mas, con lo que se tendría un desbalance que finalmente desencadene una menor generación de citoquinas y factores de crecimiento, lo que se asocia a una menor migración y proliferación celular. Por otra parte, se tendría además un cese en la inhibición de factores de transcripción involucrados con la apoptosis. De este modo, se tendría finalmente el menor número de vasos observado. Estos resultados podrían permitir plantear futuras aplicaciones terapéuticas para este fármaco en diversas patologías que cursan con angiogénesis (dermatológicas, reumatológicas), o bien incentivar su uso como factor protector (en el caso de la patología tumoral), sin embargo, su administración debe ser supervisada por el profesional a cargo, ya que en la actualidad se sabe que su uso asociado a fármacos tales como inhibidores de la enzima convertidora de angiotensina (IECA) o antagonistas del receptor de angiotensina II (ARA II) puede ser deletéreo a largo plazo en pacientes de alto riesgo cardiovascular, tales como portadores de hipertensión arterial y diabetes concomitante con enfermedad renal crónica moderada o grave (Parving et al., 2012), o con antecedentes de síndrome coronario agudo reciente (Scirica et al., 2010).

Respecto al enalapril, un IECA, se obtuvo un efecto proangiogénico, el que solo es significativo al administrarse en alta concentración. Se ha documentado la relación entre enalapril y angiogénesis, identificándolo como un regulador tanto negativo (Kim et al., 2009) como positivo (Sanchez de Miguel et al., 2008), en modelos experimentales. Nuevamente se señalan las diferencias metodológicas al compararlos con este estudio. Una posible explicación para los resultados obtenidos podría encontrarse en una propiedad de la ACE no relacionada con el SRAA: esta enzima cataliza no solo la conversión de angiotensina I en angiotensina II, sino también la degradación de bradiquininas en péptidos inactivos. Las bradiquininas son péptidos fisiológicos producidos a partir de precursores llamados quininógenos por acción de enzimas llamadas calicreínas, presentes en diversos sitios, tales como el páncreas, próstata o piel. Existen dos receptores para bradiquininas, B1 y B2, siendo este último el más importante en condiciones fisiológicas (Yazawa et al., 2011), mientras que B1 se expresa en condiciones patológicas tales como inflamación o daño tisular. En relación con la angiogénesis, la unión de bradiquininas a sus receptores, principalmente B2, estimula la acción de la enzima óxido nítrico sintetasa endotelial (eNOS), y con ello, la liberación de óxido nítrico (NO), molécula que favorece tanto la secreción de VEGF como la expresión del VEFGR-2 (Li et al., 2008). Por lo tanto, el aumento de la angiogénesis observada en los huevos tratados con enalapril se debería a su acción inhibitoria de la ACE, resultando una acumulación de bradiquininas y una mayor migración y proliferación de células endoteliales por acción del NO.

El modelo de MAC fue elegido por ser económico, no invasivo, fácil de mantener en un ambiente esterilizado, no requerir períodos prolongados de tiempo, presentar una alta tasa de sobrevida (en esta experiencia, superior al $80 \%$ ) y ser fácilmente reproducible. Además, al trabajar sobre organismos con un sistema inmune inmaduro, se descarta la posibilidad de una reacción de rechazo, ya que la instalación de los discos de metilcelulosa y la instilación de fármacos se realizan al décimo día de desarrollo, mientras que los linfocitos $\mathrm{B}$, primeras células inmunes en aparecer, no son detectados antes del día 11 (Janse \& Jeurissen, 1991). Entre sus desventajas, se tiene la presencia de un fenómeno inflamatorio inespecífico asociado a la instalación del filtro, que produce un aumento en el número de vasos sanguíneos locales que pueden amplificar falsamente el efecto angiogénico observado. Sin embargo, este fenómeno se da en todos los sujetos de estudio, por lo que se elimina un posible sesgo. Por otra parte, el aumento en el número de vasos sanguíneos puede deberse en realidad a reordenamiento de vasos sanguíneos ya presentes, y no a neoformación. Nuevamente, la ocurrencia de este proceso en todos los sujetos ayuda a limitar el posible sesgo. Otra dificultad la constituía la instilación de enalapril, fármaco ingerido por el ser humano como profármaco, a diferencia de aliskireno, que se administra como principio activo. Sin embargo, en el embrión de pollo, ya en la primera semana se tiene un sistema digestivo funcional, con un hígado capaz de metabolizar el profármaco administrado, tal como sucede en el ser humano.

$\mathrm{Al}$ elegir las concentraciones utilizadas para cada fármaco, se tuvo como referencia las concentraciones aproximadas de cada fármaco al ser ingeridas dentro de sus respectivos rangos terapéuticos en la práctica clínica 
habitual en humanos. Si bien no es posible que el modelo de MAC replique con exactitud la ingesta de humanos adultos, se espera que las dosis administradas se acerquen a las utilizadas en la práctica terapéutica. El uso de solo dos concentraciones no permite establecer patrón alguno, sea éste lineal, exponencial, en "U", o de otro tipo, que relacione dosis y efecto. Sin embargo, éste no constituía un objetivo del presente trabajo, por lo que se requieren nuevos estudios destinados a esta finalidad.
En suma, el presente trabajo confirma la hipótesis planteada respecto al efecto antiangiogénico de aliskireno en un modelo de fácil acceso que ha demostrado ser simple, replicable y válido. Los resultados obtenidos abren la puerta a nuevas líneas de investigación que permitan dilucidar, entre otros aspectos, los mecanismos subyacentes y las proyecciones que la interacción entre aliskireno y angiogénesis pueda ofrecer en relación a la terapéutica en seres humanos.

DE LA FUENTE, E. R.; ROSAS, C. C.; FUENZALIDA, B. M.; RODRÍGUEZ, B. H. ; ROA, I. \& LEMUS, A. D. Effect of aliskiren on angiogenesis in chick embryo chorioallantoic membrane (CAM) assay. Int. J. Morphol., 34(3):1191-1196, 2016.

SUMMARY: Angiogenesis is the formation of new blood vessels from pre-existing ones. Antiangiogenic effect of angiotensin receptor blockers has been reported, however, the relationship between direct renin inhibitors and angiogenesis has not been well described. To assess the antiangiogenic effect of aliskiren, a direct renin inhibitor, on chick embryo chorioallantoic membrane (CAM) assay. Aliskiren and enalapril were instilled in different concentrations and compared. In serial histological sections, the number of blood vessels per $9000 \mu \mathrm{m}^{2}$ area under observation through optical microscope using maximum zoom, was registered. Statistical analysis using Anova and Tukey test in order to show possible differences, was performed. Receptors treated with aliskiren presented lower vascular density, which was statistically significant when a higher concentration was administered. High concentrations of aliskiren have an antiangiogenic effect on CAM assay. This finding means further studies are needed, because of the usefulness direct renin inhibitors could have. These results, also, might enhance the possibility of using aliskiren for regulating angiogenesis in the context of nontransmissible chronic diseases.

KEY WORDS: Angiogenesis; Chorioallantoic membrane; Aliskiren; Enalapril.

\section{REFERENCIAS BIBLIOGRÁFICAS}

Arvelo, F. \& Cotte, C. Metaloproteasas en la progresión tumoral. Revisión. Invest. Clín., 47(2):185-205, 2006.

Bates, D. O. Vascular endothelial growth factors and vascular permeability. Cardiovasc. Res., 87(2):262-71, 2010.

Calnan, D. R. \& Brunet, A. The FoxO code. Oncogene, 27(16):2276-88, 2008.

Chang, T. T.; Wu, T. C.; Huang, P. H.; Lin, C. P.; Chen, J. S.; Lin, L. Y.; Lin, S. J. \& Chen, J. W. Direct renin inhibition with aliskiren improves ischemia-induced neovasculogenesis in diabetic animals via the SDF-1 related mechanism. PLoS One, 10(8):e0136627, 2015.

Desjarlais, M.; Dussault, S.; Dhahri, W.; Mathieu, R. \& Rivard, A. Direct renin inhibition with aliskiren improves ischemiainduced neovascularization: blood pressure-independent effect. Atherosclerosis, 242(2):450-60, 2015.

Fernández, J. G.; Rodríguez, D. A.; Valenzuela, M.; Calderon, C.; Urzúa, U.; Munroe, D.; Rosas, C.; Lemus, D.; Díaz, N.; Wright, M.; Leyton, L.; Tapia, J. \& Quest, A. F. Survivin expression promotes VEGF-induced tumor angiogenesis via PI3K/Akt enhanced b-catenin/Tcf-Lef dependent transcription. Mol. Cancer, 13:209, 2014.
Ferrão, F. M.; Lara, L. S. \& Lowe, J. Renin-angiotensin system in the kidney: What is new? World J. Nephrol., 3(3):64-76, 2014.

Ferrara, N. \& Kerbel, R. S. Angiogenesis as a therapeutic target. Nature, 438(7070):967-74, 2005.

Gavalas, N. G.; Liontos, M.; Trachana, S. P.; Bagratuni, T.; Arapinis, C.; Liacos, C.; Dimopoulos, M. A. \& Bamias, A. Angiogenesisrelated pathways in the pathogenesis of ovarian cancer. Int. $J$. Mol. Sci., 14(8):15885-909, 2013.

Goel, H. L. \& Mercurio, A. M. VEGF targets the tumour cell. Nat. Rev. Cancer, 13(12):871-82, 2013.

Harding, J. W.; Cook, V. I.; Miller-Wing, A. V.; Hanesworth, J. M.; Sardinia, M. F.; Hall, K. L.; Stobb, J. W.; Swanson, G. N.; Coleman, J. K. M.; Wright, J. W. \& Harding, E. C. Identification of an AII(3-8) [AIV] binding site in guinea pig hippocampus. Brain Res., 583(1-2):340-3, 1992.

Janse, E. M. \& Jeurissen, S. H. Ontogeny and function of two nonlymphoid cell populations in the chicken embryo. Immunobiology, 182(5):472-81, 1991.

Kim, H. W.; Kim, J. L.; Lee, H. K.; Hur, D. Y.; Yun, I. H. \& Kim, S. D. Enalapril alters expression of key growth factors in experi- 
mental diabetic retinopathy. Curr. Eye Res., 34(11):976-87, 2009.

Li, P.; Kondo, T.; Numaguchi, Y.; Kobayashi, K.; Aoki, M.; Inoue, N.; Okumura, K. \& Murohara, T. Role of bradykinin, nitric oxide, and angiotensin II type 2 receptor in imidapril-induced angiogenesis. Hypertension, 51(2):252-8, 2008.

Michel, S. P.; Medina, M. E.; Lemus, A. D. \& Sinning, O. M. Actividad angiogénica comparada de líquido cefalorraquídeo de pacientes con tumores primarios del sistema nervioso central. Rev. A. N. A. C. E. M., 5(1):12-6, 2011.

Morales Olivas, F. J. \& Yago, L. E. Aliskiren: el primer inhibidor directo de la renina introducido en terapéutica. Rev. Esp. Cardiol. Supl., 9(A):41-8, 2009.

Parving, H. H.; Brenner, B. M.; McMurray, J. J.; de Zeeuw, D.; Haffner, S. M.; Solomon, S. D.; Chaturvedi, N.; Persson, F.; Desai, A. S.; Nicolaides, M.; Richard, A.; Xiang, Z.; Brunel, P.; Pfeffer, M. A. \& ALTITUDE Investigators. Cardiorenal end points in a trial of aliskiren for type 2 diabetes. N. Engl. J. Med., 367(23):2204-13, 2012.

Passos-Silva, D. G.; Verano-Braga, T. \& Santos, R. A. Angiotensin(1-7): beyond the cardio-renal actions. Clin. Sci. (Lond.), 124(7):443-56, 2013.

Pepper, M. S. Transforming growth factor-beta: vasculogenesis, angiogenesis, and vessel wall integrity. Cytokine Growth Factor Rev., 8(1):21-43, 1997.

Pöss, J.; Werner, C.; Lorenz, D.; Gensch, C.; Böhm, M. \& Laufs, $\mathrm{U}$. The renin inhibitor aliskiren upregulates pro-angiogenic cells and reduces atherogenesis in mice. Basic Res. Cardiol., 105(6):725-35, 2010.

Rosas, C.; Sinning, M.; Ferreira, A.; Fuenzalida, M. \& Lemus, D. Celecoxib decreases growth and angiogenesis and promotes apoptosis in a tumor cell line resistant to chemotherapy. Biol. Res., 47(1):27, 2014.

Rusai, K.; Jianxing, C.; Schneider, R.; Struijker-Boudier, H.; Lutz, J.; Heemann, U. \& Baumann, M. Renin inhibition mitigates anti-angiogenesis in spontaneously hypertensive rats. $J$. Hypertens., 29(2):266-72, 2011.

Sanchez de Miguel, L.; Neysari, S.; Jakob, S.; Petrimpol, M.; Butz, N.; Banfi, A.; Zaugg, C. E.; Humar, R. \& Battegay, E. J. B2kinin receptor plays a key role in B1-, angiotensin converting enzyme inhibitor-, and vascular endothelial growth factorstimulated in vitro angiogenesis in the hypoxic mouse heart. Cardiovasc. Res., 80(1):106-13, 2008.

Santos, R. A.; Simoes e Silva, A. C.; Maric, C.; Silva, D. M.; Machado, R. P.; de Buhr, I.; Heringer-Walther, S.; Pinheiro, S. V.; Lopes, M. T.; Bader, M.; Mendes, E. P.; Lemos, V. S.; Campagnole-Santos, M. J.; Schultheiss, H. P.; Speth, R. \& Walther, T. Angiotensin-(1-7) is an endogenous ligand for the
G protein-coupled receptor Mas. Proc. Natl. Acad. Sci. U. S. A., 100(14):8258-63, 2003.

Santulli, G. Angiogenesis: Insights From a Systematic Overview. New York, Nova Science Publisher Inc., 2013.

Scirica, B. M.; Morrow, D. A.; Bode, C.; Ruzyllo, W.; Ruda, M.; Oude Ophuis, A. J.; Lopez-Sendon, J.; Swedberg, K.; Ogorek, M.; Rifai, N.; Lukashevich, V.; Maboudian, M.; Cannon, C. P.; McCabe, C. H. \& Braunwald, E. Patients with acute coronary syndromes and elevated levels of natriuretic peptides: the results of the AVANT GARDE-TIMI 43 Trial. Eur. Heart J., 31(16):1993-2005, 2010.

Schmidt, T. \& Carmeliet, P. Blood-vessel formation: Bridges that guide and unite. Nature, 465(7299):697-9, 2010.

Toledo, V.; Ramírez, G.; Valck, C.; López, N.; Ribeiro, C. H.; Maldonado, I.; Aguilar, L.; Lemus, D. \& Ferreira, A. Comparative in vivo antiangiogenic effects of calreticulin from Trypanosoma cruzi and Homo sapiens sapiens. Biol. Res., 43(3):287-9, 2010.

Vickers, C.; Hales, P.; Kaushik, V.; Dick, L.; Gavin, J.; Tang, J.; Godbout, K.; Parsons, T.; Baronas, E.; Hsieh, F.; Acton, S.; Patane, M.; Nichols, A. \& Tummino, P. Hydrolysis of biological peptides by human angiotensin-converting enzyme-related carboxypeptidase. J. Biol. Chem., 277(17):14838-43, 2002.

Yazawa, H.; Miyachi, M.; Furukawa, M.; Takahashi, K.; Takatsu, M.; Tsuboi, K.; Ohtake, M.; Murase, T.; Hattori, T.; Kato, Y.; Murohara, T. \& Nagata, K. Angiotensin-converting enzyme inhibition promotes coronary angiogenesis in the failing heart of Dahl salt-sensitive hypertensive rats. J. Card. Fail., 17(12):1041-50, 2011.

Dirección para correspondencia:

Raúl de la Fuente Espinoza

Programa de Anatomía y Biología del Desarrollo

Facultad de Medicina

Universidad de Chile

Santiago

CHILE

E-mail: rauldelaes@gmail.com

Recibido : 09-05-2016

Aceptado: 23-08-2016 